\title{
MIR846 and MIR842 comprise a cistronic MIRNA pair that is regulated by abscisic acid by alternative splicing in roots of Arabidopsis
}

\author{
Fan Jia and Christopher D. Rock ${ }^{*}$ \\ Department of Biological Sciences, Texas Tech University, Lubbock, TX 79409-3131
}

\section{Abstract}

MicroRNAs (miRNAs) are 21-nucleotide long endogenous small RNAs that regulate gene expression through post-transcriptional or transcriptional gene silencing (PTGS/TGS) and/or translational inhibition. miRNAs can arise from the "exon" of a MIRNA gene, from an intron (e.g. mirtrons in animals), or from the antisense strand of a protein coding gene (natural antisense microRNAs, nat-miRNAs). Here we demonstrate that two functionally related miRNAs, miR842 and miR846, arise from the same transcription unit but from alternate splicing isoforms. miR846 is expressed only from Isoform 1 while in Isoforms 2 and -3 , a part of pre-miR846 containing the miRNA* sequence is included in the intron. The splicing of the intron truncates the pre-MIRNA and disrupts the expression of the mature miR846.. We name this novel phenomenon splicingregulated miRNA. Abscisic acid (ABA) is shown to mediate the alternative splicing event by reducing the functional Isoform 1 and increasing the non-functional Isoform3, thus repressing the expression of miR846 concomitant with accumulation of an ABA-inducible target jacalin At5g28520 mRNA, whose cleavage was shown by modified $5^{\prime}$-RACE. This regulation shows the functional importance of splicing-regulated miRNA and suggests possible mechanisms for altered ABA response phenotypes of miRNA biogenesis mutants. A. lyrata-MIR842 and Aly-MIR846 have conserved genomic arrangements with $A$. thaliana and candidate target jacalins, similar primary transcript structures and intron processing, and better miRNA-miRNA* pairings, suggesting that the interactions between ABA, MIR842, MIR846 and jacalins are similar in A. lyrata. Together, splicing-regulated miRNAs, nat-miRNAs/inc-miRNAs and mirtrons illustrate the complexity of MIRNA genes, the importance of introns in the biogenesis and regulation of miRNAs, and raise questions about the processes and molecular mechanisms that drive MIRNA evolution.

\section{Keywords}

alternative splicing; microRNA; root; abscisic acid; plant development; pri-miRNA

\section{INTRODUCTION}

MicroRNAs (miRNAs) are approximately 21-nucleotide (nt) long endogenous small RNAs processed from primary transcripts (pri-miRNAs). Pri-miRNAs are transcribed from genomic loci (MIRNA genes) by Pol II and carry typical $5^{\prime}$ cap and $3^{\prime}$ poly-A tail structures (Lee et al. 2004; Xie et al. 2005). A pri-miRNA is cleaved into a shorter piece (a premiRNA) comprising a stem-loop by RNase III family members (Drosha in animals and Dicer-Like [DCL] proteins in plants) (Lee et al. 2003; Kurihara and Watanabe 2004). This pre-miRNA is further processed by Dicers, in association with accessory proteins such as

\footnotetext{
*To whom correspondence should be addressed: chris.rock@ttu.edu. Phone: (806)742-3722 Ext 271.
} 
DICER-ㅌNDING proteins (e.g. DRB1/HYPONASTIC LEAVES1), TOUGH, and DAWDLE, a Forkhead domain-containing protein, into a 21 nt duplex containing a $5^{\prime}$ phosphate group and two nt 3' overhangs (Vazquez et al. 2004; Yu et al. 2008; Ren et al. 2012). Interestingly, many of these factors in miRNA biogenesis also affect intron splicing, including ABH1, SERRATE and SICKLE, a proline-rich protein (Kim et al. 2008; Laubinger et al. 2008; Zhan et al. 2012). After processing by Dicers, one strand from the duplex, the mature 'guide' miRNA, is then preferentially loaded into the miRNA induced silencing complex (miRISC) containing an Argonaute (e.g. AGO1, or AGO7 for miR390) (Fahlgren et al. 2006), whereas the other strand of the duplex (miRNA*) is typically degraded. The mature miRNA guides the miRISC to a target mRNA based on Watson-Crick sequence complementarity and leads predominantly to translational inhibition in animals and mRNA cleavage in plants (Jones-Rhoades et al. 2006; Brodersen et al. 2008; Bartel 2009).

Besides the canonical biogenesis pathway, miRNAs are also generated by other mechanisms. Intronic miRNAs arise from introns, and a few cases are described in plants. A prime example in Arabidopsis is miR838, which is produced from intron 14 of the DCL1 mRNA. This intronic miR838 presumably enables a self-regulatory mechanism that helps maintain DCL1 homeostasis (Rajagopalan et al. 2006). Another Arabidopsis intronic miRNA, miR400, has recently been shown to be repressed by heat stress by affecting its alterative splicing (Yan et al. 2012). Most human miRNAs are encoded in introns (Gregory et al. 2004). It has been shown that in animals, Drosha cropping of intronic miRNAs and splicing of the intron can work independently, thereby enabling both miRNA biogenesis and protein synthesis from a single primary transcript (Kim and Kim 2007). How intronic miRNAs are generated in plants is not known. Natural antisense miRNAs (nat-miRNAs) arise from the antisense strand of protein-coding genes which are also their targets. NatmiRNAs contain introns in their stem-loop which disrupt the extensive sequence complementarity to their targets. These introns are crucial to the function of nat-miRNAs and are evolutionarily conserved (Lu et al. 2008). An artificial intron-interrupted MIRNA gene (inc-miRNA) which contained an engineered intron in its stem-loop was shown to be functional in Caenorhabditis elegans (Zhang et al. 2011), suggesting similar mechanisms of miRNA splicing might exist in animals as in plants. Both intronic miRNAs and nat-miRNAs still require canonical miRNA machinery to produce the pre-miRNAs. On the contrary, mirtrons, which are also produced from introns in animals, rely on the splicosome and exosome for the production of pre-miRNAs (Okamura et al. 2007; Ruby et al. 2007; Zhu et al. 2008; Flynt et al. 2010). Candidates of plant mirtrons have been reported recently (Zhu et al. 2008; Meng and Shao 2012), and there is also genetic evidence that the exosome plays a role in miRNA biogenesis in plants (Chekanova et al. 2007). Despite such complexity and the importance of miRNA biogenesis, study is obstructed by the low abundance of primary transcripts, which are rapidly turned into pre-miRNAs (Lobbes et al. 2006; Song et al. 2007). Moreover, pri-miRNAs can often have multiple transcription start sites (TSSs) and poly-adenylation signals (Szarzynska et al. 2009), which suggests a low evolutionary selective pressure outside the stem-loop structure for MIRNA genes.

In animals, MIRNA genes are largely encoded in polycistrons, and these polycistronic miRNAs often target the same mRNA (Altuvia et al. 2005; Kim and Nam 2006). This situation is presumably due to the mode of action in that miRNAs predominantly repress translation by binding to the 3'UTR region of the target mRNA in animals, and it often requires multiple miRNAs to repress a single mRNA (Bartel 2009). Thereby, clustered miRNAs on a single transcription unit results in efficient regulation of the target. On the other hand, plant miRNAs function mainly through target mRNA slicing by the miRISC, in which case a single miRNA efficiently represses its target (Jones-Rhoades et al. 2006). In accordance with this, most plant miRNAs are transcribed from independent transcription 
units. However, recent research has shown that plants also have polycistronic transcripts containing different miRNAs, and similar to observations in animals, these polycistronic miRNAs target genes from the same family. However, the functional importance of these polycistronic miRNAs in plants remains unclear (Merchan et al. 2009).

miRNAs are important regulators of development and stress responses, evidenced by the phenotypes of miRNA processing mutants which have developmental defects such as low fertility and abnormal morphology (Bohmert et al. 1998; Jacobsen et al. 1999; Chen et al. 2002). Beside miRNAs, plant hormones also play important roles in growth and development. The interactions between auxin and several miRNAs [miR160 (Mallory et al. 2005; Wang et al. 2005; Gutierrez et al. 2009), miR164 (Guo et al. 2005), miR167 (Gutierrez et al. 2009; Kinoshita 2012), miR390 (Allen et al. 2005; Williams et al. 2005; Yoon et al. 2010) and miR393 (Xie et al. 2000; Navarro et al. 2006)] and their targets to regulate root development and biotic stress responses have been studied in detail, and provide a paradigm for understanding signaling crosstalk in plants (Meng et al. 2010). Another less understood example is the association between abscisic acid (ABA) and miRNAs. Intriguingly, mutations in several key components in the miRNA pathway are hypersensitive to ABA. These mutants include drb1/hyponastic leaves1 (hyl-1), dcl-1, ABA hypersensitive1/cap-binding-protein80 (abh1), and small RNA methyltransferase hen $1(\mathrm{Lu}$ and Fedoroff 2000; Hugouvieux et al. 2001; Gregory et al. 2008; Zhang et al. 2008). ABA has also been shown to regulate accumulation of a few miRNAs including miR159 and miR319, which function to repress MYB transcription factors that are positive regulators in ABA signaling during seed germination (Reyes and Chua 2007; Kim et al. 2008). Taken together, these findings provide compelling evidence of interactions between ABA signaling and miRNA pathways and raise questions about the molecular mechanisms of $\mathrm{ABA}$ action in post-transcriptional regulation.

In this study, we show that two functionally related miRNAs unique to the Arabidopsis species $A$. thaliana and $A$. lyrata, miR842 and miR846, arise from the same transcription unit but alternate splicing isoforms. miR846 is expressed only from Isoform1 while in Isoforms 2 and -3 a part of pre-miR846 containing the miR846* sequence is included in the intron, preventing the formation of a stem-loop structure when spliced. We name this novel phenomenon splicing-regulated miRNA. Exogenous application of ABA to seedlings mediates the alternative splicing event by reducing the functional Isoform 1 and increasing the non-functional Isoform 3 and thus represses the expression of miR846 concomitant with accumulation of the At5g28520/jacalin mRNA. The interaction between ABA, MIR846 and MIR842 provides an example of ABA-miRNA interaction and shows the functional importance of splicing-regulated miRNA. Furthermore, we find MIR842 and MIR846 in A. lyrata and show that they have conserved genomic arrangements, similar primary transcript structures, better miRNA-miRNA* parings, as well as target jacalin homologs. It suggests that the interactions between ABA, MIR842, MIR846 and jacalins may function in A. lyrata. Together, splicing-regulated miRNAs, nat-miRNAs/inc-miRNAs and mirtrons illustrate the complexity of MIRNA genes, the importance of introns in the biogenesis and regulation of miRNAs, and raise questions about the processes and molecular mechanisms that drive MIRNA evolution.

\section{RESULTS}

\section{miR842 and miR846 are processed from the same transcription unit}

AT-MIR842 and AT-MIR846 are oriented sequentially on the Watson strand of Chromosome 1, but despite mapping less than 200 bp away in the genome, a previous attempt failed to amplify any common transcript (Merchan et al. 2009). We designed a pair of primers (Pair A, Fig. 1A) covering the entire $610 \mathrm{bp}$ stem-loop regions of both MIR842 
and MIR846 and performed reverse transcription PCR (RT-PCR) to detect possible common transcripts in total RNA extracts of 10-d old seedlings. A $372 \mathrm{bp}$ fragment was amplified (Fig. 1B) and sequenced. The $\sim 240$ bp difference comparing the predicted genomic amplicon to cloned cDNA revealed the presence of an intron, with characteristic intron junction sequences $5^{\prime}$-GU...AG-3'. This intron mapped downstream of the MIR842 hairpin and within the stem-loop of MIR846 such that the intron-spliced cDNA fragment included only the mature miR846 leg of pre-miR846 hairpin. This result suggested this species of mRNA could produce only mature miR842 from the spliced intron species, but not miR846.

In order to further characterize expression of the locus, standard 5' Rapid Amplification of cDNA Ends ( $\left.5^{\prime} \mathrm{RACE}\right)$ was then performed independently with nested reverse strand genespecific primers for MIR842 or MIR846 (Fig. 1A; open and solid arrowheads, respectively). Two amplicons were cloned and sequenced; when taken together with the results from primer Pair A amplicon, these results revealed the existence of two splice variants which have the same transcription start site (TSS) $\sim 100 \mathrm{bp}$ upstream of a shared $5^{\prime}$ splice site (Fig. 1A). We then performed $3^{\prime}$ RACE, and results revealed variable $3^{\prime}$ termination sites within a $150 \mathrm{bp}$ region downstream of pre-miR846 (data not shown).

Using a novel primer pair covering the whole region (Pair B, Fig. 1A), RT-PCR was performed and three transcripts of the sizes $578 \mathrm{bp}, 482 \mathrm{bp}$ and $316 \mathrm{bp}$ were amplified (Fig. $1 \mathrm{~A}, \mathrm{C}$, and D). The $482 \mathrm{bp}$ transcript, designated Isoform1, was the most abundant based on band intensities. It contained the complete pre-miR846 in the second "exon" and the complete pre-miR842 in the first intron. The 578 bp transcript, designated Isoform 2 , contained the complete pre-mir842 in exon 2 . It corresponded to the initial amplicon characterized by primer Pair A (Fig. 1A and B), where part of pre-mir846 including the miR846* sequence was spliced out as an intron, which would preclude the production of miR846. The $316 \mathrm{bp}$ transcript, designated Isoform3, contained the first and third exons of Isoform 2 and skipped exon 2 . These results prove that miR842 and miR846 are produced from the same transcription unit. However, unlike polycistronic miRNAs, the mature miRNAs arise from different splicing products. miR846 is expressed only from Isoform 1 whereas mir842 arises from the stable product Isoform2, or possibly from the introns produced during splicing of Isoforms 1 and -3 .

\section{MIR842 and MIR846 are expressed predominantly in roots}

To better understand the expression of both miRNAs, we constructed a T-DNA expression vector comprising presumed promoter of MIR842 and MIR846 (a $\sim 1 \mathrm{~kb}$ fragment between the $3^{\prime}$ UTR of the upstream gene AT1G61215 and the shared TSS of MIR842 and MIR846) fused with the bacterial reporter gene uidA (GUS; $\beta$-glucuronidase) in pBI121. Histochemical GUS staining of 10-d old transgenic seedlings carrying the ProMIR842_MIR846:GUS reporter construct showed strong GUS activity in roots and leaf primordia but not in mature leaves (8 out of 10 independent lines) (Fig. 2). GUS staining did not show any difference between wild-type and Pro-MIR842_MIR846:GUS transgenic plants in rosette leaves, inflorescence or siliques, where some background (non-transgenic) GUS activity could be seen in vascular tissue, floral organs, and at the junction between silique and petiole (Supplemental Fig. S1). These results are supported by small RNA deep sequencing datasets which show that the normalized reads per million (rpm) of AGO1associated miR846 in different tissues were 69.6 (flowers), 458 (leaves), 2130 (roots) and 1170 (whole seedlings) and that of miR842 were 4.4 (flowers), 11.6 (leaves), 75.8 (roots) and 76.7 (whole seedlings) (Wang et al. 2011, see their Table S4). These deep sequencing data also correlate with the observed relative high abundance of Isoform1 (482 bp band, expressing miR846) versus Isoform2 (578 bp band, expressing miR842 but not miR846) by RT-PCR (Fig. 1C). 
miR846 accumulation is under ABA regulation and correlates with alternative splicing of pri-miR846

A small RNA blot was performed to examine the expression of mature miR842 and miR846 using total RNAs from leaves or roots of 10-d old seedlings (Fig. 3A). The observation that both miRNAs were expressed mainly in roots verifies the promoter analysis and AGO1associated small RNAseq results (Fig. 2; see above). Interestingly, both miR842 and miR846 abundances were repressed by increasing amounts of exogenous ABA treatment of roots, with the strongest ( $35 \%$ reduction for both miRNAs) effect observed after $3 \mathrm{hr}$ exposure to ABA (Fig. 3A). The expression of pri-miR846 (Isoform1) was then quantified with semi-quantitative RT-PCR using primer Pair C (Fig. 1A). It showed that $3 \mathrm{hr}$ treatment of $10 \mu \mathrm{M}$ ABA repressed pri-miR846 levels with a $\sim 30 \%$ reduction (Fig. 3B) which is consistent with the small RNA blot result.

Moreover, when amplified with primer Pair B (Fig. 1A) to see the relative expression levels of the three Isoforms, RT-PCR results showed that without exogenous ABA treatment, the non-functional Isoform 3 was below the detection limit and Isoform1, which generates miR846, was expressed at a high level. In response to increasing concentrations of exogenous ABA, Isoform1 expression was reduced while Isoform3 levels increased (Fig. 3C). Isoform 2 , which can produce miR842, was not detected in these experiments, due to the smaller number of cycles used in the PCR (34 instead of 38) to avoid saturation.

Alternatively, real-time PCR was used to examine the response of Isoform 2 to ABA treatment using a primer pair specific to the stem-loop of MIR842 as well as to Isoform 2 (Fig. 1D, open arrows). Random primers were used in the reverse transcription for the detection of both Isoform 2 and pre-MIR842 (Fig. 3D). By contrast, oligo-dT primer was used for detecting only Isoform 2 (Fig. 3E). The results showed a significant decrease in abundance in response to $3 \mathrm{hr}$ treatment with $10 \mu \mathrm{M}$ ABA for Isoform 2 plus pre-MIR842 (Fig. 3D) but not for Isoform 2 itself (Fig 3E) in 10-day old seedlings. Therefore the reduced abundance shown in Fig. 3D was due to reduced abundance of pre-MIR842, which is consistent with the small RNA blot result.

Taken together, these results demonstrate that ABA represses expression of both miR842 and miR 846 by affecting the splicing of the primary transcript and changing the relative amounts of the Isoforms, from Isoform 1 that produces miR846 in the absence of ABA to Isoform 3 that produces no functional miR846, and miR842 only if the spliced intron were processed by a Dicer, in the presence of ABA.

\section{A potential target $A T 5 G 28520$ of miR842 and miR846 is highly up-regulated by ABA in roots}

Both miR842 and miR846 target several jacalin lectin family members containing mannosebinding lectin domains (InterPro IPR001229) (Rajagopalan et al. 2006; Fahlgren et al. 2007). The Arabidopsis genome encodes 112 mannose-binding domain-containing genes (http://www.ebi.ac.uk). An online tool "psRNATarget" (http://plantgrn.noble.org/ psRNATarget/) (Dai and Zhao, 2011) was used to identify possible targets of miR842 and miR846 (Supplemental Table S1). Consistent with previous reports, miR842 and miR846 both were predicted to target several jacalin lectin family members with mismatch scores less than 2.5. One of these targets, AT5G28520, was highly up-regulated by ABA and abiotic stresses in roots according to Arabidopsis eFP browser (http://bar.utoronto.ca/) (Supplemental Fig. S2) (Winter et al. 2007). RNA blot analysis of 10-d old seedlings showed that the ABA induction of AT5G28520 was specific in roots in a time- and ABA dosage- dependent manner (Fig. 4A, B). 
The complementary sites to miR842 and miR846 are at the $5^{\prime}$ end of AT5G28520, each with 2 mismatches and one G:U-wobble (Fig. 4C). Modified 5' RACE (Llave et al., 2002) using various gene-specific reverse primers mapping downstream of both target site on total RNAs from ABA-treated seedlings detected a single cleavage event mapping to the miR846 target site, at an atypical position between nt 6 and 7 in the absence, but not presence, of exogenous ABA (Fig. 4D). No cleavage event was detected within a predicted miR842 target site. To rule out the possibility that all target mRNA molecules were simultaneously cleaved at both positions hence only the fragment corresponding to the $3^{\prime}$ miR846 site had been detected, a second modified $5^{\prime}$-RACE experiment was performed using a reverse primer that was downstream of miR842 target site but upstream of miR846 target site (AT5g28520_192R, Supplemental Table S2); again, no cleavage event was detected (data not shown).

\section{A similar genomic arrangement for miR842 and miR846 exists in A. lyrata}

Recent studies revealed that miR842 and miR846 exist in $A$. lyrata, a close relative of $A$. thaliana (Fahlgren et al. 2010; Ma et al. 2010). A BLAST search with $A$. thaliana miR842 and miR846 precursor sequences against the $A$. lyrata genomic sequence (http://genome.jgipsf.org/Araly1/Araly1.home.html) identified a genomic region that contained both pre-AlymiR842 and pre-Aly-miR846 hairpins. Alignments between $A$. thaliana and A. lyrata MIR842/MIR846 genomic sequences showed high similarity, especially in the pre-miRNA regions. Noticeably, all GT...AG donor-acceptor sequences were conserved in both species as well (Supplemental Fig. S3). A total of three substitutions were found in the star legs of A. lyrata pre-miRNAs (Fig. 5A). One of them converted an A:U pair in $A$. thaliana to a G:U wobble in $A$. lyrata; the other two substitutions closed up gaps present in the $A$. thaliana miRNA-miRNA* duplexes, which is consistent with purifying selection operating on MIR842 and MIR846 to maintain function in these species.

To confirm the expression of MIR842 and MIR846 in A. lyrata, a primer pair flanking both pre-MIR842 and pre-MIR846 (ALmiR842/6_F and ALmiR842/6_R1, Fig. 5C arrows) was used in RT-PCR and a band of predicted size was amplified from total RNAs of 10-day old A. lyrata seedlings but not from the negative control (Fig. 5B). Furthermore, one A. lyrata expressed sequence tag (EST) (XM002888052.1, Fig. 5C) was found mapping to this region. RT-PCR with a second primer pair covering the EST (AlmiR842/6_F and ALmiR842/6_R2, Fig 5C arrows) produced three bands (Fig. 5B). Sequencing of PCR products showed the upper band (1,314 bp) covered the whole genomic region; the middle band $(1,164 \mathrm{bp})$ had an intron close to the $3^{\prime}$ end which did not affect the expression of either miRNA; whereas the lower band ( $356 \mathrm{bp}$ ) contained an additional intron that included the complete stem-loop of miR846, similar to Isoform 1 in A. thaliana.

To search for the jacalin lectin targets of Aly-miR842 and Aly-miR846, homologs of AT5G28520 were searched in A. lyrata using TBLASTN. Mature miR842 or miR846 sequences were then used to BLAST against the search results. Two jacalin homologs were found to carry complementary sites to both miR842 and miR846 in A. lyrata (Fig 5D). Six additional potential targets of miR846 were also found in A. lyrata (Supplemental Fig. S4).

\section{DISCUSSION}

\section{miR842 and miR846 are produced from splicing alternatives of the same transcription unit}

In this study, we found that miR842 and miR846 are produced from the same transcription unit but different splicing isoforms (Fig. 1D). miR846 is expressed only from Isoform1 because in Isoforms 2 and -3 , a part of pre-miR846 containing the miRNA* sequence is included in the intron, preventing the formation of a stem-loop structure after splicing. 
miR842 can be expressed from the exon of Isoform2, or possibly from the introns of Isoforms 1 and -3 , contingent upon the spliced introns not being degraded before DCL processing. The fact that both mature miR842 (Fig. 3A) and pre-MIR842, but not Isoform 2, are down-regulated by ABA (Fig. 3D and E) suggests that Isoform 2 is less likely to express the majority of miR842. We postulate that miR842 is mainly produced from the intron of Isoform 1 because it is the only isoform that is down regulated by ABA. The potential functionality of intronic miR842 is supported by the existence of miR838, which is also an intronic miRNA (Rajagopalan et al. 2006) and by the fact that in animals, the cropping of such intronic miRNAs and the splicing of the intron can happen independently on the same transcript (Kim and Kim 2007). It is also interesting to note that maximum expression of the Pro-MIR842_MIR846:GUS reporter in the root stele and zone of differentiation (Fig. 2A, D) corresponds with expression of the ABA-inducible marker Dc3 (Rock and Sun, 2005).

\section{miR846 is a splicing-regulated miRNA}

Nat-miRNAs and the artificial inc-miRNAs have introns in their stem-loop sequences and by splicing they are able to produce functional miRNAs (Lu et al. 2008; Zhang et al. 2011) (Supplemental Fig. S5B). Here we present a different mechanism whereby a functional miR846 is disrupted by an intron (Fig. 1D). The expression of this miRNA can thereby be regulated through alternative splicing to switch between functional and non-functional Isoforms as we have shown in Fig. 1D and 3C. We therefore name the process 'splicingregulated miRNA'. A nat-miRNA or inc-miRNA could be theoretically classed as splicingregulated miRNAs if shown to be regulated in a similar way; i.e. evidence of retention (nonfunctional isoform) versus splicing away the intron in the stem-loop to effect expression of a functional isoform. Furthermore, mirtrons in animals (Supplemental Fig. S5C) could also be regulated by alternative splicing, as the production of pre-miRNAs of mirtrons relies on the flanking intron-exon junctions (Okamura et al. 2007; Ruby et al. 2007; Zhu et al. 2008; Flynt et al. 2010). Together, splicing-regulated miRNAs, nat-miRNAs/inc-miRNAs and mirtrons illustrate the complexity of MIRNA genes and the importance of introns in the biogenesis and function of miRNAs.

\section{ABA represses miR846 by affecting its alternative splicing}

ABA is known to affect the steady-state levels of some miRNAs in Arabidopsis. However, despite claims of ABA-regulated miRNAs (Sunkar and Zhu 2004; Reyes and Chua 2007; Kim et al. 2008; Jia et al. 2009; Li et al. 2012), little has been done to study how miRNA expression might be controlled by ABA. Our findings demonstrate that ABA can regulate miR842 and miR846 post- transcriptionally by affecting the alternative splicing of primiRNA transcripts (Fig. 3C). We speculate the advantage of affecting alternative splicing of pri-miRNAs over transcriptional inhibition by ABA would be that the rapid change of the relative amounts of splicing isoforms could be modulated rather than changes in transcription rates per se. This would allow Arabidopsis to rapidly turn on and off the expression of miRs 842 and 846 and would in turn allow a rapid strong induction of the target $A t 5 g 28520$ in the presence of ABA (Fig. 4). Given the fact that many plant miRNAs have intron structures and splicing isoforms (e.g. MIR156C, MIR160A, MIR160B, $M I R 166 B$ ) (Xie et al. 2005; Szarzynska et al. 2009) and that there are plant mirtrons which require correct splicing to be expressed (Zhu et al. 2008; Meng and Shao 2012), this mechanism of regulation of miRNA abundance may function beyond MIR846. Exactly how this mechanism is related to the expression of $A T 5 G 28520$ jacalin (Fig. 4A and B) and potentially other cases remains to be elucidated. However, reports of miRNAs associated with antisense transcription (Luo et al., 2009) and DNA methylation (Chellappan et al., 2010) of targets in Arabidopsis may provide some clues. 


\section{The function of miR846 and miR842}

miR842 and miR846 are predicted to target several jacalin lectin family members and one of them, AT5G28520 is expressed specifically in roots in the presence of exogenous ABA (Fig. $4 \mathrm{~A}$ and B). miR842 and miR846 are also enriched in roots (Wang et al., 2011) and their abundances are affected by ABA (Fig. 3A). Using modified $5^{\prime}$-RACE, a cleavage event was found within miR846 complementary site between position 7 and 8 right at the point of an A-G mismatch (Fig. 4C). Conventionally, miRNAs result in RISC cleavage of mRNAs between position 10 and 11. Although the observed cleavage position is non-canonical for typical miR846, a variant which starts at nt -3 of miR846 would serve as a canonical guide to the mapped cleavage event (Fig. 4C; Supplemental Fig. S6).

miRNA variants that originate from the same precursor are called isomiRs, which can have variations in the $5^{\prime}$ or $3^{\prime}$ end, or in their lengths (Morin et al. 2008; Neilsen et al. 2012). IsomiRs with variable $5^{\prime}$ ends have been discovered in both plants (Jeong et al. 2011; Jeong and Green 2012) and animals (Landgraf et al. 2007; Ruby et al. 2007; Azuma-Mukai et al. 2008; Lee et al. 2010). Despite the lower abundance comparing to the canonical miRNA, some IsomiRs show tissue- or condition- specific expression and are biologically meaningful (Fernandez-Valverde et al. 2010; Jeong et al. 2011). It is noteworthy that quantitative analysis of read abundances documented in miRBase (www.mirbase.org) (Kozomara and Griffith-Jones, 2011) show 7\% of miR846 species in seedling (45.8RPM out of 641.8RPM; GEO dataset GSM118375) (Rajagopalan et al., 2006; Mi et al. 2008) begin at variable positions upstream of the published mature sequence. The actual isomiR which starts at nt -3 of miR846 that would serve as a canonical guide to the mapped cleavage event (Fig. 4C; Supplemental Fig. S6) is detected in roots (1 read in LS1, early meristematic tissue; 3 reads in LS3, elongation zone) (Breakfield et al. 2012). Furthermore, both miR842 and miR846 pair perfectly with AT5G28520 in the 2-7 nt 'seed' regions (Fig. 4C) (Bartel 2009). Given the fact that there is widespread translational inhibition by plant miRNAs (Brodersen et al. 2008), it is worth considering whether miR842 and miR846 regulate AT5G28520 through translational inhibition. However, until these models are experimentally verified, the functional importance of miR842 and miR846 in ABAregulated target jacalin At5g28520 expression remains unclear.

Evolution of miRNAs is not well understood, yet the AtMIR842/MIR846 cluster is a cognate for a modified model of $M I R N A$ evolution (Allen et al. 2004) from inverted duplication of target genes (Rajagopalan et al. 2006). The fact that many predicted jacalin targets have a miR842 binding site upstream of miR846, and that the genomic organization of the MIR842/MIR846 cluster is in the same orientation as the targets (miR842 upstream), suggests a common evolutionary origin of these targets and MIRNAs. If there were dual cleavage events by miR842 and miR846, then the 'two hit' model of biogenesis of secondary siRNAs by the PTGS pathway could be in play (Axtell et al. 2006), yet there is no such evidence that any jacalin targets produce siRNAs, supporting our claim that only miR846 likely cleaves At5g28520 mRNA. Routing of miRNA targets into siRNA pathways is likely under negative purifying selection and/or tightly regulated. When it does occur, it reinforces the silencing of transcripts from the miRNA target locus, or it might have some function conceivably to expand the range of target repression or network crosstalk to related family members that interact with the siRNAs (Cuperus et al. 2011). Whether other members of the jacalin family of miR842/miR846 targets are regulated by ABA, other crosstalk or homeostatic feedback mechanisms, or factors affecting differential cleavage remains to be determined. 


\section{ABA, MIR842, MIR846 and jacalin target regulation may function in A. lyrata}

miR842 and miR846 are non-conserved miRNAs, i.e. recently evolved and found in narrow clades and subject to relatively frequent birth and death, which raise questions of their functional significance (Rajagopalan et al. 2006; Fahlgren et al. 2007). The finding of a conserved genomic arrangement of MIR842 and MIR846 (Supplemental Fig. S3) and better base pairings between miRNA and miRNA* legs in $A$. lyrata supports that both miRNAs are active and under purifying selection to maintain the hairpin structure (Figure 5A). Using RT-PCR, we confirmed that MIR842 and MIR846 are expressed in A. lyrata (Fig. 5B and C). Interestingly, our RT-PCR results show that as in $A$. thaliana, different splicing isoforms for MIR842 and MIR846 also exist in A. lyrata. One of these isoforms (lower band, Fig. 5C) contains the stem loop of miR842 in an exon and the stem loop of miR846 in an intron, which is very similar to Isoform 2 in $A$. thaliana. Furthermore, we were able to find jacalin homologs which have miR842 and miR846 complementary sites in A. lyrata(Fig. 5D). Taken together, the conserved genomic arrangements, similar primary transcript structures, better miRNA-miRNA* parings, and the presence of target jacalin homologs in A. lyrata suggest that the interactions between ABA, MIR842, MIR846 and jacalins may function in A. lyrata. We were able to find extended homology (E > 10 ${ }^{-11}$; data not shown) in Brassica rapa and Thellungiella halophila (now T. salsuginea) to At-MIR846 hairpin (www.phytozome.org), however the structural significance of the homologies could not be established by folding these sequences into stable hairpins, suggesting that MIR842 and MIR846 do not exist in these genera whose tribes (Brassicae and Eutremeae, respectively) diverged from each other and the Arabidae an estimated 40 million years ago (Bailey et al., 2006). Three outstanding aspects of the evolution of MIR842 and MIR846 are the exaptation of $\mathrm{ABA}$ signaling into the regulatory network involving its potential target jacalin lectin AT5G28520, these effectors' functions in ABA control of root development (Vartanian et al. 1994) and insight into the evolution of stress tolerance, since Thellungiella lives in extreme salt, cold, and freezing conditions and is a model system for study efficient mobilization of resources in poor or degraded soils (Inan et al., 2004).

\section{MATERIALS AND METHODS}

\section{Plasmid construction}

For the promoter of MIR842 and MIR846, a $\sim 1 \mathrm{~kb}$ sequence between the $3^{\prime}$ UTR of the upstream gene At1g61215 and the shared TSS of MIR842 and MIR846 were PCR-amplified using PrimeSTAR HS DNA polymerase (TAKARA, Shiga, Japan) and inserted between the Hind III and BamHI sites in pBI121, replacing the 35S promoter to drive uidA GUS reporter gene expression. Oligonucleotide primers were designed using using 'Perlprimer' (http:// perlprimer.sourceforge.net/) and synthesized commercially (Sigma, St. Louis MO). A list of primers used in the study is provided in Supplemental Table S2.

\section{Plant material and growth conditions}

Arabidopsis thaliana Col-0 (Arabidopsis Biological Resource Center, Ohio State University; http://abrc.osu.edu/, stock\# CS60000) and Arabidopsis lyrata (ABRC stock\# CS22696) seeds were soaked in water and kept in the dark at $4^{\circ} \mathrm{C}$ for $3 \mathrm{~d}$ before transferring to soil. The growth condition was $21^{\circ} \mathrm{C}$ with a $16 \mathrm{hr}$ light and $8 \mathrm{hr}$ dark cycle. For promoter analysis, Col-0 plants were transformed with Agrobacterium tumefaciens strain GV3101 carrying Pro-MIR842_MIR846:GUS constructs using the floral dip method (Zhang et al., 2006).

For ABA treatments, seeds were sown on plates containing $0.5 x$ Murashige \& Skoog salts (Research Products International, Mt. Prospect, IL), $1 \%$ sucrose and $0.5 \%$ phytagel (SIGMA-ALDRICH, St. Louis, MO). Plates were kept in the dark at $4^{\circ} \mathrm{C}$ for $3 \mathrm{~d}$ before 
transferring into a growth chamber. The growth condition was $21^{\circ} \mathrm{C}$ with continuous light. Plates were kept in the chamber for $10 \mathrm{~d}$ before spraying with ABA solutions. ABA $(( \pm)-$ Abscisic acid, 98\%; ACROS Organics, New Jersey) solutions were diluted with water from a $100 \mu \mathrm{M}$ stock in $70 \%$ ethanol. The same amount of $70 \%$ ethanol was added to water to spray the control group.

\section{$\beta$-Glucuronidase GUS assays}

Plant samples were developed at $37^{\circ} \mathrm{C}$ for $12 \mathrm{hr}$ with a $1 \mathrm{mM}$ solution of the indigogenic GUS substrate 5-bromo-4-chloro-3-indolyl b-D-glucu-ronide (X-Gluc; Rose Scientific, Edmonton, Alberta, Canada) in $50 \mathrm{mM} \mathrm{KH}_{2} \mathrm{PO}_{4}$ (pH 7.0), $0.1 \mathrm{mM}$ EDTA, $0.5 \mathrm{mM}$ ferricyanide, $0.5 \mathrm{mM}$ ferrocyanide, $0.05 \%$ sodium azide, $0.1 \%$ Triton $\mathrm{X}-100$. After staining overnight, samples were immersed in $70 \%$ ethanol overnight. Samples were then mounted in $30 \%$ glycerol on microscope slides for taking pictures with a dissecting microscope, or were imaged with an Olympus BX41 light microscope for high magnification detailed imaging using QCapture software (v2.68.6, Silicon Graphics, Fremont, CA).

\section{Analysis of gene expression with PCR}

Total RNA from roots or leaves of 10-d old seedlings, or from whole seedlings, was extracted with Iso-RNA Lysis Reagent (5 PRIME, Gaithersburg, MD).

For RT-PCR, $4 \mu \mathrm{g}$ total RNA was digested with RQ1 RNase-free DNase (Promega, Madison, WI) and reverse transcribed by MMLV reverse transcriptase (Promega) with Anchored Oligo-dT (ABgene, Surrey, UK). A cycle number of 38 was used for PCR experiments in Figure 1B and 1C. For quantitative analysis of experiments shown in Figure 3B and 3C, PCR reactions were performed with 30, 34, 38 or 40 cycles and the results at 34 cycles were analyzed to avoid saturation artifacts. Band intensities were quantified with ImageJ software (http://rsbweb.nih.gov/ij/index.html).

For real-time PCR, cDNA was prepared the same as for RT-PCR except that random primers (Promega) were used during reverse transcription. Real-time PCRs were performed using Absolute SYBR Green Fluorescein (Thermo-Scientific, Surrey, UK) mixed with ROX Passive reference Dye (Bio-Rad, Hercules, CA) according to the supplier's specification and were run on an Applied Biosystems AB7000 instrument with default settings. The results were analyzed with affiliated software.

RACE experiments were performed with GeneRacer Kit (Invitrogen, Carlsbad, CA) according to the manufacturer's specification.

\section{RNA blot hybridization}

For high molecular-weight northern hybridization, samples of $10 \mu \mathrm{g}$ total RNA were resolved ona $1.2 \%$ denaturing agarose gel and blotted to a Hybond- $\mathrm{N}^{+}$membrane (GE Healthcare, Piscataway, NJ) according to the supplier's protocol. At5g28520 template was amplified using primers "At5g28520CDS_F" and "At5g28520CDS" from a cDNA library and was eluted after excision of the band from an agarose gel. Probes were synthesized using Random Primer DNA Labeling Kit Ver.2 (TAKARA, Shiga, Japan) with [a- $\left.{ }^{32} \mathrm{P}\right]-$ dCTP (PerkinElmer, Waltham, MA).

For small RNA northern hybridization, $120 \mu \mathrm{g}$ total RNA for each sample was resolved on a $17 \%$ polyacrylamide/7 M urea/ $0.5 \mathrm{x}$ TBE gel and blotted to a Hybond- $\mathrm{N}^{+}$membrane (GE Healthcare, Piscataway, NJ) using a semi-dry transferring system. To generate probes, oligonucleotides with the exact complementary sequence to miR842 or miR846 were endlabeled with $\left[\gamma^{-}{ }^{32} \mathrm{P}\right]$-ATP (PerkinElmer, Waltham, MA) and T4 polynucleotide kinase (New 
England Biolabs, Ipswich, MA). For miR846, a Locked Nucleic Acid (LNA) oligonucleotide (Valoczi et al., 2004) was synthesized (Exicon, www.exiqon.com; Woburn MA) (see Supplemental Table S2).

For both high-molecular weight and small RNA northern, hybridization was carried out with the PerfectHyb Plus hybridization buffer (SIGMA-ALDRICH, Saint Louis, MO) according to the manufacturer's instructions. A storage phosphor screen (GE Healthcare, Piscataway, NJ) was used for autoradiography and it was scanned using a Storm 860 PhosphorImager (GE Healthcare). Band intensities were quanti ed using the ImageQuant TL software (v2003, GE Healthcare).

\section{Bioinformatic analysis}

Potential targets of At-miR842 or At-miR846 were indentified with "psRNATarget" (http:// plantgrn.noble.org/psRNATarget/) (Dai and Zhao, 2011). Potential targets of Aly-miR842 or Aly-miR846 were obtained by BLASTing miR842 or miR846 sequence against Expressed Sequence Tags (ESTs) of $A$. lyrata (GenBank, www.ncbi.nlm.nih.gov). Aly-MIR842 and Aly-MIR846 genomic sequence was obtained by BLASTing Ath-MIR842 and Ath-MIR846 genomic sequence against $A$. lytrata genome (http://genome.jgi-psf.org/Araly1/ Araly1.home.html). The alignment was performed with T-Coffee software (www.tcoffee.org).

For the analysis of miR846 variants, datasets for root smRNAs were downloaded from the NCBI Sequence Read Archive (SRA, http://www.ncbi.nlm.nih.gov/Traces/sra) (Wheeler et al. 2008). The accessions used were SRR218085 (LS1), SRR218086 (LS2), SRR218087 (LS3) and SRR218088 (LS4) (Breakfield et al. 2012). Raw data were converted to fastq format using SRA tool kit (http://www.ncbi.nlm.nih.gov/Traces/sra/sra.cgi?view=software). The $3^{\prime}$ adapter sequence was clipped and reads shorter then 13 nt were discarded using FASTX-tool kit (http://hannonlab.cshl.edu/fastx_toolkit/index.html). Alignment was then performed using bowtie (http://bowtie-bio.sourceforge.net/index.shtml) (Langmead et al. 2009).

\section{Supplementary Material}

Refer to Web version on PubMed Central for supplementary material.

\section{Acknowledgments}

The authors thank Qingjun Luo, Amandeep Mittal and Yingwen Jiang for helpful discussions, and the TTU High Performance Computing Center for use of the Hrothgar cluster. This work was supported by the National Institutes of Health grant GM077245 to C.D.R, and a Texas Tech University AT\&T Chancellor's Fellowship to F.J. The funders had no role in the study design, in the collection, analysis, or interpretation of data, or in the writing of the manuscript or decision to submit the manuscript for publication.

\section{References}

Allen E, Xie ZX, Gustafson AM, Sung GH, Spatafora JW, Carrington JC. Evolution of microRNA genes by inverted duplication of target gene sequences in Arabidopsis thaliana. Nature Genetics. 2004; 36:1282-1290. [PubMed: 15565108]

Allen E, Xie Z, Gustafson AM, Carrington JC. microRNA-directed phasing during trans-acting siRNA biogenesis in plants. Cell. 2005; 121:207-221. [PubMed: 15851028]

Altuvia Y, Landgraf P, Lithwick G, Elefant N, Pfeffer S, Aravin A, Brownstein MJ, Tuschl T, Margalit H. Clustering and conservation patterns of human microRNAs. Nucleic Acids Res. 2005; 33:2697-2706. [PubMed: 15891114] 
Axtell MJ, Jan C, Rajagopalan R, Bartel DP. A two-hit trigger for siRNA biogenesis in plants. Cell. 2006; 127:565-577. [PubMed: 17081978]

Azuma-Mukai A, Oguri H, Mituyama T, Qian ZR, Asai K, Siomi H, Siomi MC. Characterization of endogenous human Argonautes and their miRNA partners in RNA silencing. Proc Natl Acad Sci U S A. 2008; 105:7964-7969. [PubMed: 18524951]

Bailey CD, Koch MA, Mayer M, Mummenhoff K, O’Kane SL, Warwick SI, Windham MD, AlShehbaz IA. Toward a global phylogeny of the Brassicaceae. Mol Biol Evol. 2006; 23:2142-2160. [PubMed: 16916944]

Bartel DP. MicroRNAs: target recognition and regulatory functions. Cell. 2009; 136:215-233. [PubMed: 19167326]

Cuperus JT, Fahlgren N, Carrington JC. Evolution and functional diversification of MIRNA genes. Plant Cell. 2011; 23:431-442. [PubMed: 21317375]

Langmead B, Trapnell C, Pop M, Salzberg SL. Ultrafast and memory-efficient alignment of short DNA sequences to the human genome. Genome Biology. 2009; 10:R25. [PubMed: 19261174]

Bohmert K, Camus I, Bellini C, Bouchez D, Caboche M, Benning C. AGO1 defines a novel locus of Arabidopsis controlling leaf development. EMBO J. 1998; 17:170-180. [PubMed: 9427751]

Breakfield NW, Corcoran DL, Petricka JJ, Shen J, Sae-Seaw J, Rubio-Somoza I, Weigel D, Ohler U, Benfey PN. High-resolution experimental and computational profiling of tissue-specific known and novel miRNAs in Arabidopsis. Genome Res. 2012; 22:163-176. [PubMed: 21940835]

Brodersen P, Sakvarelidze-Achard L, Bruun-Rasmussen M, Dunoyer P, Yamamoto YY, Sieburth L, Voinnet O. Widespread translational inhibition by plant miRNAs and siRNAs. Science. 2008; 320:1185-1190. [PubMed: 18483398]

Chekanova JA, Gregory BD, Reverdatto SV, Chen H, Kumar R, Hooker T, Yazaki J, Li P, Skiba N, Peng Q, et al. Genome-wide high-resolution mapping of exosome substrates reveals hidden features in the Arabidopsis transcriptome. Cell. 2007; 131:1340-1353. [PubMed: 18160042]

Chellappan P, Xia J, Zhou X, Gao S, Zhang X, Coutino G, Vazquez F, Zhang W, Jin H. siRNAs from miRNA sites mediate DNA methylation of target genes. Nucleic Acids Res. 2010; 38:6883-6894. [PubMed: 20621980]

Chen X, Liu J, Cheng Y, Jia D. HEN1 functions pleiotropically in Arabidopsis development and acts in C function in the flower. Development. 2002; 129:1085-1094. [PubMed: 11874905]

Dai X, Zhao PX. psRNATarget: a plant small RNA target analysis server. Nucleic Acids Res. 2011; 39:W155-159. [PubMed: 21622958]

Fahlgren N, Howell MD, Kasschau KD, Chapman EJ, Sullivan CM, Cumbie JS, Givan SA, Law TF, Grant SR, Dangl JL, et al. High-throughput sequencing of Arabidopsis microRNAs: evidence for frequent birth and death of MIRNA genes. PLoS ONE. 2007; 2:e219. [PubMed: 17299599]

Fahlgren N, Jogdeo S, Kasschau KD, Sullivan CM, Chapman EJ, Laubinger S, Smith LM, Dasenko M, Givan SA, Weigel D, et al. MicroRNA gene evolution in Arabidopsis lyrata and Arabidopsis thaliana. Plant Cell. 2010; 22:1074-1089. [PubMed: 20407027]

Fahlgren N, Montgomery TA, Howell MD, Allen E, Dvorak SK, Alexander AL, Carrington JC. Regulation of AUXIN RESPONSE FACTOR3 by TAS3 ta-siRNA affects developmental timing and patterning in Arabidopsis. Curr Biol. 2006; 16:939-944. [PubMed: 16682356]

Fernandez-Valverde SL, Taft RJ, Mattick JS. Dynamic isomiR regulation in Drosophila development. RNA. 2010; 16:1881-1888. [PubMed: 20805289]

Finkelstein R, Gampala SS, Lynch TJ, Thomas TL, Rock CD. Redundant and distinct functions of the ABA response loci $A B A$-INSENSITIVE(ABI)5 and ABRE-BINDING FACTOR (ABF)3. Plant Mol Biol. 2005; 59:253-267. [PubMed: 16247556]

Flynt AS, Greimann JC, Chung WJ, Lima CD, Lai EC. MicroRNA biogenesis via splicing and exosome-mediated trimming in Drosophila. Mol Cell. 2010; 38:900-907. [PubMed: 20620959]

Gregory BD, O’Malley RC, Lister R, Urich MA, Tonti-Filippini J, Chen H, Millar AH, Ecker JR. A link between RNA metabolism and silencing affecting Arabidopsis development. Dev Cell. 2008; 14:854-866. [PubMed: 18486559]

Gregory RI, Yan KP, Amuthan G, Chendrimada T, Doratotaj B, Cooch N, Shiekhattar R. The Microprocessor complex mediates the genesis of microRNAs. Nature. 2004; 432:235-240. [PubMed: 15531877] 
Guo HS, Xie Q, Fei JF, Chua NH. MicroRNA directs mRNA cleavage of the transcription factor NAC1 to downregulate auxin signals for Arabidopsis lateral root development. Plant Cell. 2005; 17:1376-1386. [PubMed: 15829603]

Gutierrez L, Bussell JD, Pacurar DI, Schwambach J, Pacurar M, Bellini C. Phenotypic plasticity of adventitious rooting in Arabidopsis is controlled by complex regulation of AUXIN RESPONSE FACTOR transcripts and microRNA abundance. Plant Cell. 2009; 21:3119-3132. [PubMed: 19820192]

Hugouvieux V, Kwak JM, Schroeder JI. An mRNA cap binding protein, ABH1, modulates early abscisic acid signal transduction in Arabidopsis. Cell. 2001; 106:477-487. [PubMed: 11525733]

Inan G, Zhang Q, Li PH, Wang ZL, Cao ZY, Zhang H, Zhang CQ, Quist TM, Goodwin SM, Zhu JH, et al. Salt cress. A halophyte and cryophyte Arabidopsis relative model system and its applicability to molecular genetic analyses of growth and development of extremophiles. Plant Physiol. 2004; 135:1718-1737. [PubMed: 15247369]

Jacobsen SE, Running MP, Meyerowitz EM. Disruption of an RNA helicase/RNAse III gene in Arabidopsis causes unregulated cell division in floral meristems. Development. 1999; 126:52315243. [PubMed: 10556049]

Jeong DH, Park S, Zhai J, Gurazada SG, De Paoli E, Meyers BC, Green PJ. Massive analysis of rice small RNAs: mechanistic implications of regulated microRNAs and variants for differential target RNA cleavage. Plant Cell. 2011; 23:4185-207. [PubMed: 22158467]

Jeong DH, Green PJ. Methods for validation of miRNA sequence variants and the cleavage of their targets. Methods. 201210.1016/j.ymeth.2012.08.005

Jia X, Wang WX, Ren L, Chen QJ, Mendu V, Willcut B, Dinkins R, Tang X, Tang G. Differential and dynamic regulation of miR398 in response to ABA and salt stress in Populus tremula and Arabidopsis thaliana. Plant Mol Biol. 2009; 71:51-59. [PubMed: 19533381]

Jones-Rhoades MW, Bartel DP, Bartel B. MicroRNAs and their regulatory roles in plants. Annu Rev Plant Biol. 2006; 57:19-53. [PubMed: 16669754]

Kim S, Yang JY, Xu J, Jang IC, Prigge MJ, Chua NH. Two cap-binding proteins CBP20 and CBP80 are involved in processing primary microRNAs. Plant Cell Physiol. 2008; 49:1634-1644. [PubMed: 18829588]

Kim VN, Nam JW. Genomics of microRNA. Trends Genet. 2006; 22:165-173. [PubMed: 16446010]

Kim YK, Kim VN. Processing of intronic microRNAs. EMBO J. 2007; 26:775-783. [PubMed: 17255951]

Kinoshita N, Wang H, Kasahara H, Liu J, Macpherson C, Machida Y, Kamiya Y, Hannah MA, Chua NH. IAA-Ala Resistant3, an Evolutionarily Conserved Target of miR167, Mediates Arabidopsis Root Architecture Changes during High Osmotic Stress. Plant Cell. 201210.1105/tpc. 112.097006

Kozomara A, Griffiths-Jones S. miRBase: integrating microRNA annotation and deep-sequencing data. Nucleic Acids Res. 2011; 39:D152-D157. [PubMed: 21037258]

Kurihara Y, Watanabe Y. Arabidopsis micro-RNA biogenesis through Dicer-like 1 protein functions. Proc Natl Acad Sci U S A. 2004; 101:12753-12758. [PubMed: 15314213]

Landgraf P, Rusu M, Sheridan R, Sewer A, Iovino N, Aravin A, Pfeffer S, Rice A, Kamphorst AO, Landthaler M. A mammalian microRNA expression atlas based on small RNA library sequencing. Cell. 2007; 129:1401-1414. [PubMed: 17604727]

Laubinger S, Sachsenberg T, Zeller G, Busch W, Lohmann JU, Rätsch G, Weigel D. Dual roles of the nuclear cap-binding complex and SERRATE in pre-mRNA splicing and microRNA processing in Arabidopsis thaliana. Proc Natl Acad Sci U S A. 2008; 105:8795-8800. [PubMed: 18550839]

Lee LW, Zhang S, Etheridge A, Ma L, Martin D, Galas D, Wang K. Complexity of the microRNA repertoire revealed by next-generation sequencing. RNA. 2010; 16:2170-2180. [PubMed: 20876832]

Lee Y, Ahn C, Han J, Choi H, Kim J, Yim J, Lee J, Provost P, Radmark O, Kim S, et al. The nuclear RNase III Drosha initiates microRNA processing. Nature. 2003; 425:415-419. [PubMed: 14508493]

Lee Y, Kim M, Han J, Yeom KH, Lee S, Baek SH, Kim VN. MicroRNA genes are transcribed by RNA polymerase II. EMBO J. 2004; 23:4051-4060. [PubMed: 15372072] 
Li W, Cui X, Meng Z, Huang X, Xie Q, Wu H, Jin H, Zhang D, Liang W. Transcriptional regulation of Arabidopsis MIR168a and argonaute1 homeostasis in abscisic acid and abiotic stress responses. Plant Physiol. 2012; 158:1279-1292. [PubMed: 22247272]

Llave C, Xie ZX, Kasschau KD, Carrington JC. Cleavage of Scarecrow-like mRNA targets directed by a class of Arabidopsis miRNA. Science. 2002; 297:2053-2056. [PubMed: 12242443]

Lobbes D, Rallapalli G, Schmidt DD, Martin C, Clarke J. SERRATE: a new player on the plant microRNA scene. EMBO Rep. 2006; 7:1052-1058. [PubMed: 16977334]

Lu C, Fedoroff N. A mutation in the Arabidopsis HYL1 gene encoding a dsRNA binding protein affects responses to abscisic acid, auxin, and cytokinin. Plant Cell. 2000; 12:2351-2366. [PubMed: $11148283]$

Lu C, Jeong DH, Kulkarni K, Pillay M, Nobuta K, German R, Thatcher SR, Maher C, Zhang L, Ware $\mathrm{D}$, et al. Genome-wide analysis for discovery of rice microRNAs reveals natural antisense microRNAs (nat-miRNAs). Proc Natl Acad Sci U S A. 2008; 105:4951-4956. [PubMed: 18353984]

Luo QJ, Samanta MP, Koksal F, Janda J, Galbraith DW, Richardson CR, Ou-Yang F, Rock CD. Evidence for antisense transcription associated with microRNA target mRNAs in Arabidopsis. PLoS Genet. 2009; 5:e1000457. [PubMed: 19381263]

Ma Z, Coruh C, Axtell MJ. Arabidopsis lyrata small RNAs: transient MIRNA and small interfering RNA loci within the Arabidopsis genus. Plant Cell. 2010; 22:1090-1103. [PubMed: 20407023]

Mallory AC, Bartel DP, Bartel B. MicroRNA-directed regulation of Arabidopsis AUXIN RESPONSE FACTOR 17 is essential for proper development and modulates expression of early auxin response genes. Plant Cell. 2005; 17:1360-1375. [PubMed: 15829600]

Meng Y, Ma X, Chen D, Wu P, Chen M. MicroRNA-mediated signaling involved in plant root development. Biochem Biophys Res Commun. 2010; 393:345-349. [PubMed: 20138828]

Meng Y, Shao C. Large-scale identification of mirtrons in Arabidopsis and rice. PLoS ONE. 2012; 7:e31163. [PubMed: 22348048]

Merchan F, Boualem A, Crespi M, Frugier F. Plant polycistronic precursors containing nonhomologous microRNAs target transcripts encoding functionally related proteins. Genome Biol. 2009; 10:R136. [PubMed: 19951405]

Mi S, Cai T, Hu Y, Chen Y, Hodges E, Ni F, Wu L, Li S, Zhou H, Long C, et al. Sorting of small RNAs into Arabidopsis Argonaute complexes is directed by the $5^{\prime}$ terminal nucleotide. Cell. 2008; 133:116-127. [PubMed: 18342361]

Morin RD, O’Connor MD, Griffith M, Kuchenbauer F, Delaney A, Prabhu AL, Zhao Y, McDonald H, Zeng T, Hirst M, et al. Application of massively parallel sequencing to microRNA profiling and discovery in human embryonic stem cells. Genome Res. 2008; 18:610-621. [PubMed: 18285502]

Nagano AJ, Fukao Y, Fujiwara M, Nishimura M, Hara-Nishimura I. Antagonistic jacalin-related lectins regulate the size of ER body-type beta-glucosidase complexes in Arabidopsis thaliana. Plant Cell Physiol. 2008; 49:969-980. [PubMed: 18467340]

Nagano AJ, Matsushima R, Hara-Nishimura I. Activation of an ER-body-localized beta-glucosidase via a cytosolic binding partner in damaged tissues of Arabidopsis thaliana. Plant Cell Physiol. 2005; 46:1140-1148. [PubMed: 15919674]

Navarro L, Dunoyer P, Jay F, Arnold B, Dharmasiri N, Estelle M, Voinnet O, Jones JD. A plant miRNA contributes to antibacterial resistance by repressing auxin signaling. Science. 2006; 312:436-439. [PubMed: 16627744]

Neilsen CT, Goodall GJ, Bracken CP. IsomiRs - the overlooked repertoire in the dynamic microRNAome. Trends Genet. 201210.1016/j.tig.2012.07.005

Okamura K, Hagen JW, Duan H, Tyler DM, Lai EC. The mirtron pathway generates microRNA-class regulatory RNAs in Drosophila. Cell. 2007; 130:89-100. [PubMed: 17599402]

Rajagopalan R, Vaucheret H, Trejo J, Bartel DP. A diverse and evolutionarily fluid set of microRNAs in Arabidopsis thaliana. Genes Dev. 2006; 20:3407-3425. [PubMed: 17182867]

Ren G, Xie M, Dou Y, Zhang S, Zhang C, Yu B. Regulation of miRNA abundance by RNA binding protein TOUGH in Arabidopsis. Proc Natl Acad Sci U S A. 2012; 109:12817-12821. [PubMed: 22802657] 
Reyes JL, Chua NH. ABA induction of miR159 controls transcript levels of two MYB factors during Arabidopsis seed germination. Plant J. 2007; 49:592-606. [PubMed: 17217461]

Rock CD, Sun X. Crosstalk between ABA and auxin signaling pathways in roots of Arabidopsis thaliana (L.) Heynh. Planta. 2005; 222:98-106. [PubMed: 15889272]

Ruby JG, Jan CH, Bartel DP. Intronic microRNA precursors that bypass Drosha processing. Nature. 2007; 448:83-86. [PubMed: 17589500]

Ruby JG, Stark A, Johnston WK, Kellis M, Bartel DP, Lai EC. Evolution, biogenesis, expression, and target predictions of a substantially expanded set of Drosophila microRNAs. Genome Res. 2007; 17:1850-1864. [PubMed: 17989254]

Song L, Han MH, Lesicka J, Fedoroff N. Arabidopsis primary microRNA processing proteins HYL1 and DCL1 define a nuclear body distinct from the Cajal body. Proc Natl Acad Sci U S A. 2007; 104:5437-5442. [PubMed: 17369351]

Sunkar R, Zhu JK. Novel and stress-regulated microRNAs and other small RNAs from Arabidopsis. Plant Cell. 2004; 16:2001-2019. [PubMed: 15258262]

Szarzynska B, Sobkowiak L, Pant BD, Balazadeh S, Scheible WR, Mueller-Roeber B, Jarmolowski A, Szweykowska-Kulinska Z. Gene structures and processing of Arabidopsis thaliana HYL1dependent pri-miRNAs. Nucleic Acids Res. 2009; 37:3083-3093. [PubMed: 19304749]

Válóczi A, Hornyik C, Varga N, Burgyan J, Kauppinen S, Havelda Z. Sensitive and specific detection of microRNAs by northern blot analysis using LNA-modified oligonucleotide probes. Nucleic Acids Res. 2004; 32:e175. [PubMed: 15598818]

Vartanian N, Marcotte L, Giraudat J. Drought rhizogenesis in Arabidopsis thaliana: differential responses of hormonal mutants. Plant Physiol. 1994; 104:761-767. [PubMed: 12232124]

Vazquez F, Gasciolli V, Crete P, Vaucheret H. The nuclear dsRNA binding protein HYL1 is required for microRNA accumulation and plant development, but not posttranscriptional transgene silencing. Curr Biol. 2004; 14:346-351. [PubMed: 14972688]

Wang H, Zhang X, Liu J, Kiba T, Woo J, Ojo T, Hafner M, Tuschl T, Chua NH, Wang XJ. Deep sequencing of small RNAs specifically associated with Arabidopsis AGO1 and AGO4 uncovers new AGO functions. Plant J. 2011; 67:292-304. [PubMed: 21457371]

Wang JW, Wang LJ, Mao YB, Cai WJ, Xue HW, Chen XY. Control of root cap formation by microRNA-targeted Auxin Response Factors in Arabidopsis. Plant Cell. 2005; 17:2204-2216. [PubMed: 16006581]

Wheeler DL, Barrett T, Benson DA, Bryant SH, Canese K, Chetvernin V, Church DM, Dicuccio M, Edgar R, Federhen S, et al. Database resources of the National Center for Biotechnology Information. Nucleic Acids Res. 2008; 36:D13-21. [PubMed: 18045790]

Williams L, Carles CC, Osmont KS, Fletcher JC. A database analysis method identifies an endogenous trans-acting short-interfering RNA that targets the Arabidopsis ARF2, ARF3, and ARF4 genes. Proc Natl Acad Sci U S A. 2005; 102:9703-9708. [PubMed: 15980147]

Winter D, Vinegar B, Nahal H, Ammar R, Wilson GV, Provart NJ. An "Electronic Fluorescent Pictograph" browser for exploring and analyzing large-scale biological data sets. PLoS One. 2007; 2:e718. [PubMed: 17684564]

Xie Q, Frugis G, Colgan D, Chua NH. Arabidopsis NAC1 transduces auxin signal downstream of TIR1 to promote lateral root development. Genes Dev. 2000; 14:3024-3036. [PubMed: 11114891]

Xie Z, Allen E, Fahlgren N, Calamar A, Givan SA, Carrington JC. Expression of Arabidopsis MIRNA genes. Plant Physiol. 2005; 138:2145-2154. [PubMed: 16040653]

Yamada K, Hara-Nishimura I, Nishimura M. Unique defense strategy by the endoplasmic reticulum body in plants. Plant Cell Physiol. 2011; 52:2039-2049. [PubMed: 22102697]

Yamaji Y, Maejima K, Komatsu K, Shiraishi T, Okano Y, Himeno M, Sugawara K, Neriya Y, Minato $\mathrm{N}$, Miura C, et al. Lectin-mediated resistance impairs plant virus infection at the cellular level. Plant Cell. 2012; 24:778-793. [PubMed: 22307853]

Yan K, Liu P, Wu CA, Yang GD, Xu R, Guo QH, Huang JG, Zheng CC. Stress-nduced alternative splicing provides a mechanism for the regulation of microRNA processing in Arabidopsis thaliana. Mol Cell. 2012; 484:521-531. [PubMed: 23063528] 
Yoon EK, Yang JH, Lim J, Kim SH, Kim SK, Lee WS. Auxin regulation of the microRNA390dependent trans-acting small interfering RNA pathway in Arabidopsis lateral root development. Nucleic Acids Res. 2010; 38:1382-1391. [PubMed: 19969544]

Yu B, Bi L, Zheng B, Ji L, Chevalier D, Agarwal M, Ramachandran V, Li W, Lagrange T, Walker JC, et al. The FHA domain proteins DAWDLE in Arabidopsis and SNIP1 in humans act in small RNA biogenesis. Proc Natl Acad Sci U S A. 2008; 105:10073-10078. [PubMed: 18632581]

Zeevaart JA. Changes in the levels of abscisic acid and its metabolites in excised leaf blades of Xanthium strumarium during and after water stress. Plant Physiol. 1980; 66:672-678. [PubMed: 16661500]

Zhan X, Wang B, Li H, Liu R, Kalia RK, Zhu JK, Chinnusamy V. Arabidopsis proline-rich protein important for development and abiotic stress tolerance is involved in microRNA biogenesis. Proc Natl Acad Sci U S A. 2012; 109:18198-18203. [PubMed: 23071326]

Zhang X, Henriques R, Lin SS, Niu QW, Chua NH. Agrobacterium-mediated transformation of Arabidopsis thaliana using the floral dip method. Nat Protocols. 2006; 1:641-646.

Zhang H, Maniar JM, Fire AZ. 'Inc-miRs': functional intron-interrupted miRNA genes. Genes Dev. 2011; 25:1589-1594. [PubMed: 21828269]

Zhang JF, Yuan LJ, Shao Y, Du W, Yan DW, Lu YT. The disturbance of small RNA pathways enhanced abscisic acid response and multiple stress responses in Arabidopsis. Plant Cell Environ. 2008; 31:562-574. [PubMed: 18208512]

Zhu QH, Spriggs A, Matthew L, Fan L, Kennedy G, Gubler F, Helliwell C. A diverse set of microRNAs and microRNA-like small RNAs in developing rice grains. Genome Res. 2008; 18:1456-1465. [PubMed: 18687877] 
A

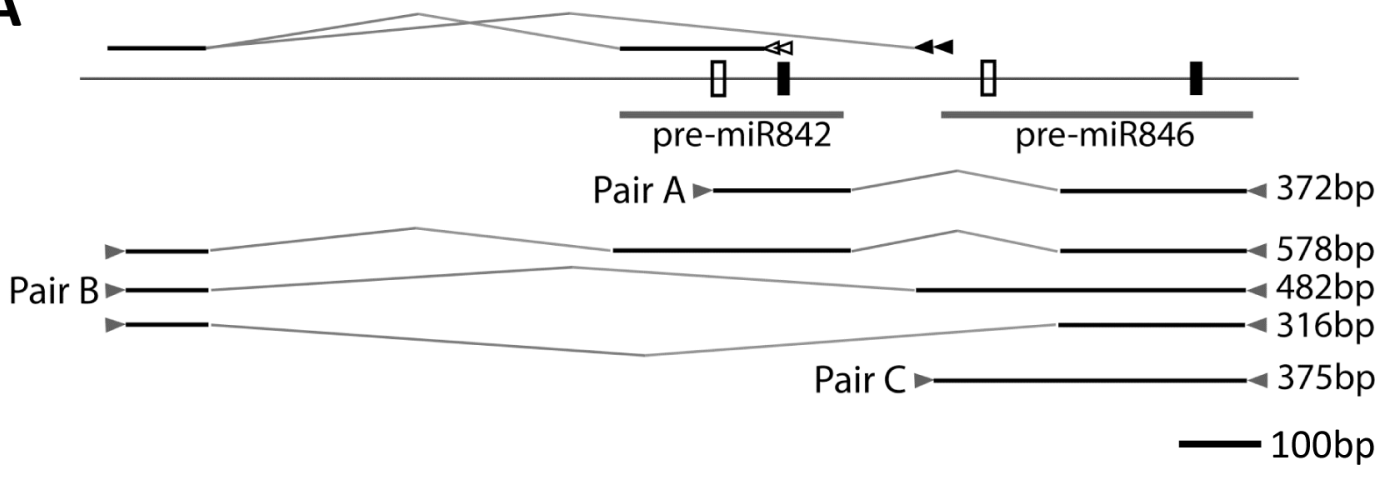

B

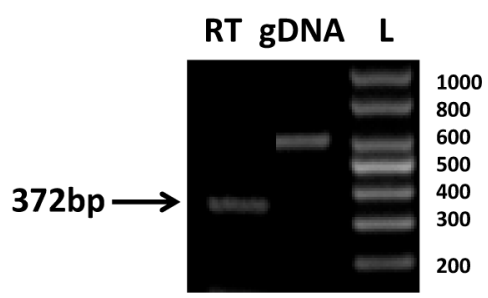

C

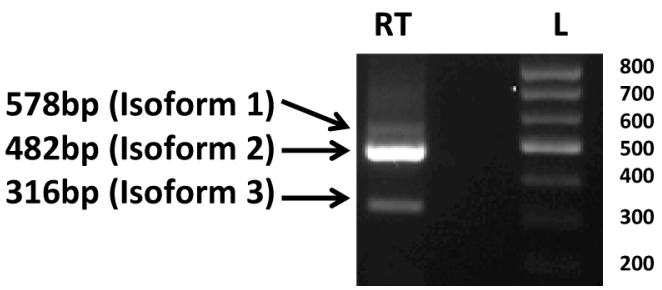

D
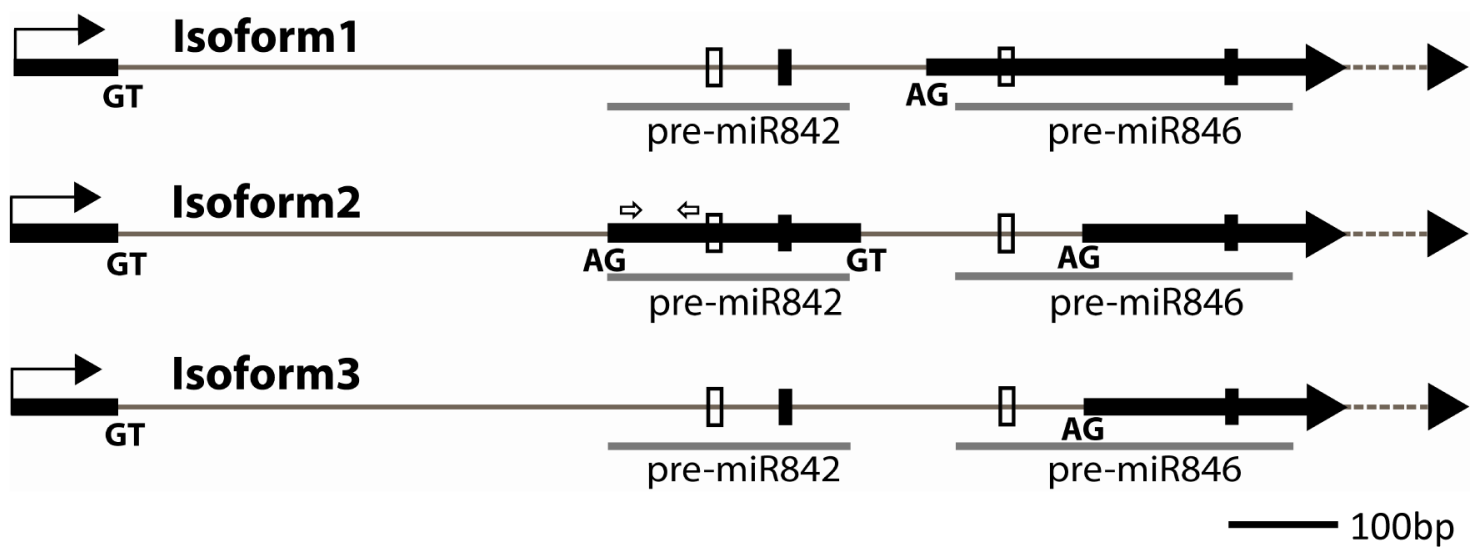

Fig. 1. miR842 and miR846 are processed from the same transcription unit (A) Genomic arrangement of pre-miR842 and pre-miR846. Thick gray lines (labels below) represent pre-miR842 or pre-miR846. Open boxes represent miRNA* and black boxes represent mature miRNA sequences. Open arrowheads represent primers used in $5^{\prime}$-RACE for pri-miR842; solid arrowheads represent primers used in $5^{\prime}$-RACE for pri-miR846; gray arrow heads represent primers used in reverse transcriptase (RT)-PCR. Black lines between the arrow heads represent exons and gray bent lines represent introns identified by RT-PCR. Scale bar, 100bp. (B) Ethidium-stained agarose gel electrophoresis of RT-PCR products using total RNA from 10-d old seedlings as template, amplified with primer Pair A. gDNA, genomic DNA control. L, DNA ladder. (C) Ethidium-stained agarose gel electrophoresis of RT-PCR product with primer Pair B. L, DNA ladder. (D) Genomic structure of Isoforms1, -, 2 and -3 . Thick black lines represent "exons," thin gray lines represent introns. Labels are as described in panel $(A)$. Black right-angle lines with arrows indicate the TSS. Broken gray lines represent variable $3^{\prime}$ termination sites. The GT..AG intron $5^{\prime}$ - and $3^{\prime}$-splice 
boundaries are shown in bold letters. Open arrows represent primers used in real-time PCR in Fig 3D and E. 

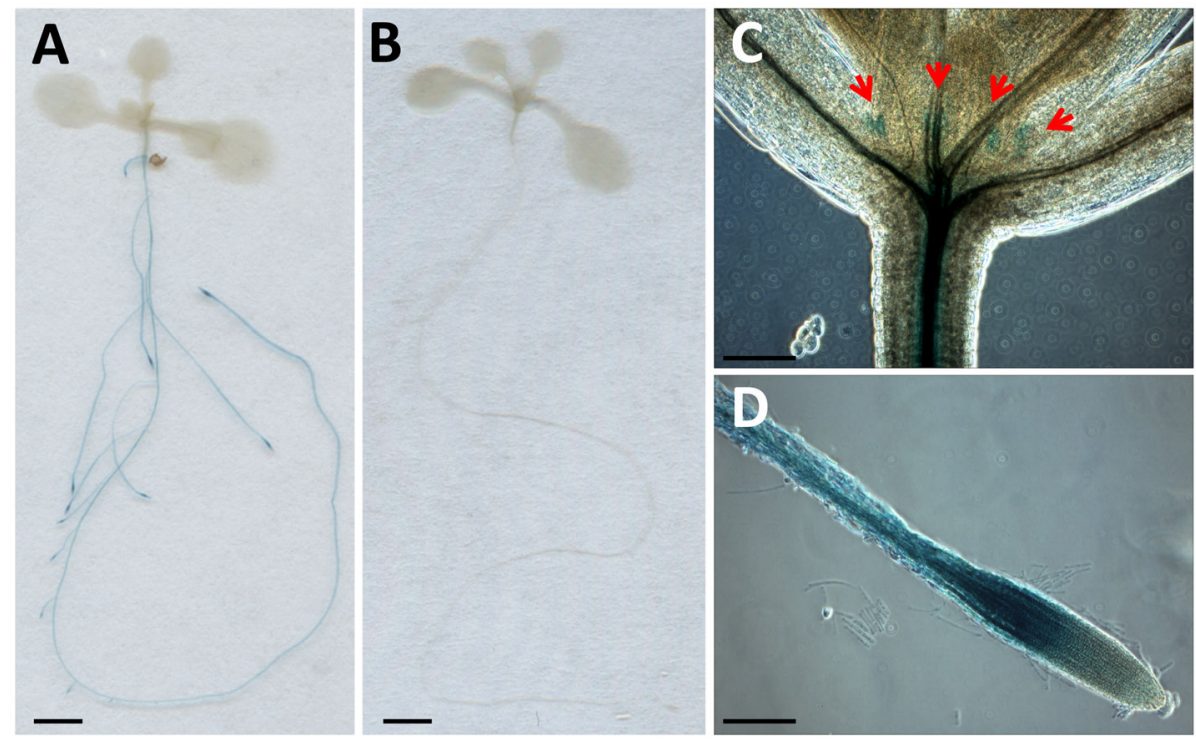

Fig. 2. Expression of GUS reporter driven by the $1 \mathrm{kbp}$ promoter of At-MIR842 and At-MIR846 (A) GUS staining of a 10-d old Pro-MIR842_MIR846:GUS transgenic seedlings, showing strong GUS expression in roots. $(B)$ GUS staining of a 10-d old Col-0 seedling. Scale bars, 2 $\mathrm{mm}$. (C) Leaf primordia-specific GUS expression in the transgenic seedlings. Red arrows point to leaf primordia. $(D)$ Strong GUS expression in the zone of elongation in root tips of the transgenic seedlings. Scale bars, $100 \mu \mathrm{m}$. 
A

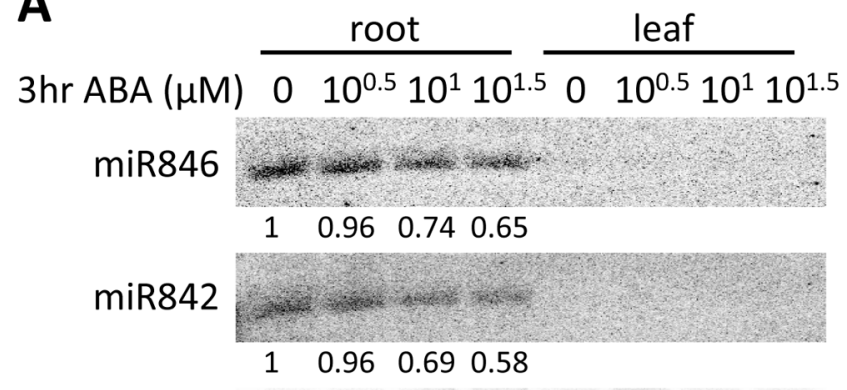

U6

C

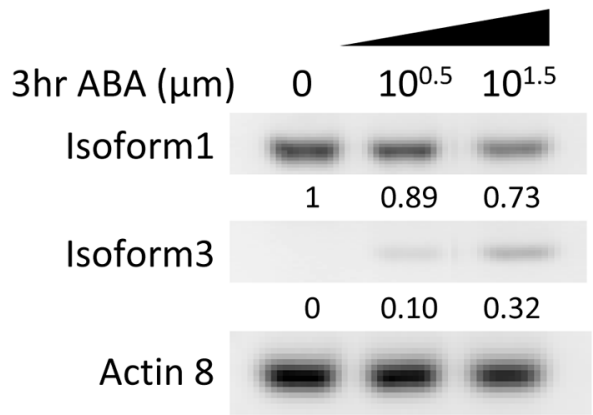

B

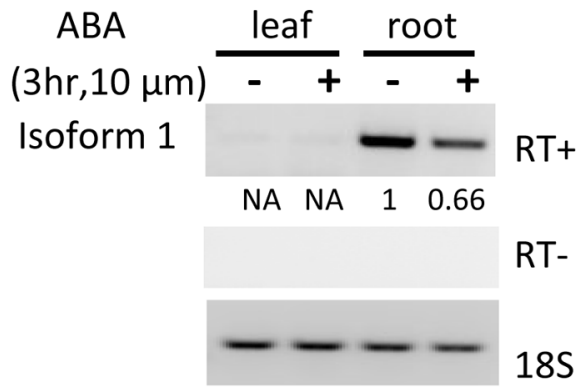

D

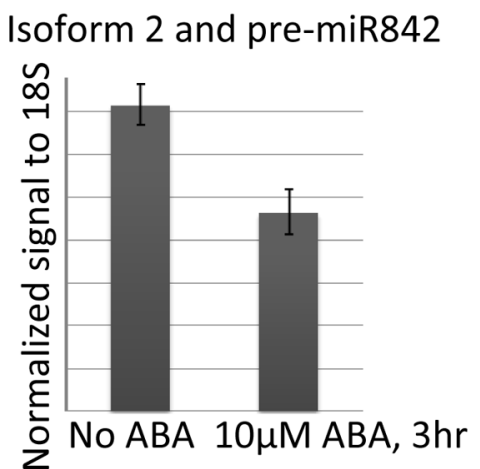

E

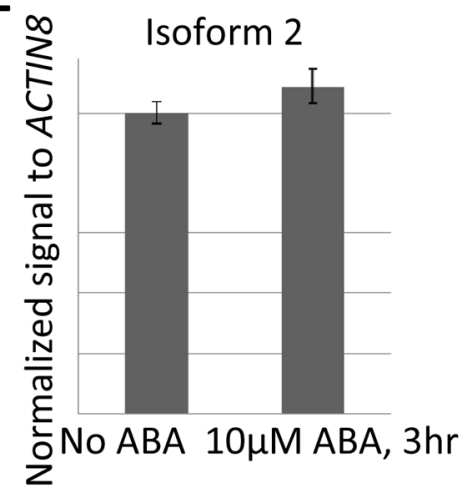

Fig. 3. miR846 and miR842 are repressed by $A B A$ in roots

(A) Small RNA blot of miR846 and miR842 in roots or leaves of 10-d old seedlings treated with increasing concentrations of ABA for $3 \mathrm{hr}$. Numbers below the bands are normalized intensities relative to zero ABA treatment, after internal normalization to U6 snRNA loading control. (B) Semi-quantitative RT-PCR with primer Pair C (see Figure 1A) using total RNA from leaves or roots of $10-\mathrm{d}$ old seedlings treated with or without $10 \mu \mathrm{M} \mathrm{ABA}$ for $3 \mathrm{hr}$. (C) Semi-quantitative RT-PCR with primer Pair B (see Figure 1A) using total RNA from roots of 10-d old seedlings treated with increasing amount of ABA for $3 \mathrm{hr}$. Numbers below the bands in $(B)$ and $(C)$ are relative intensities normalized to ACTIN8. (D) Real-time PCR result showing reduced abundance of pri-miR842 (isoform 2) and pre-miR842 in response to $3 \mathrm{hr}$ treatment of $10 \mu \mathrm{M}$ ABA in 10-day old seedlings. Signals are normalized to $18 \mathrm{~S}$. Error bar, standard error, $n=3$. (E) Real-time PCR result showing no change in the abundance of isoform 2 in response to $3 \mathrm{hr}$ treatment of $10 \mu \mathrm{M} \mathrm{ABA}$ in 10-day old seedlings. Signals are normalized to ACTIN8. Error bar, standard error, $\mathrm{n}=2$. 
A

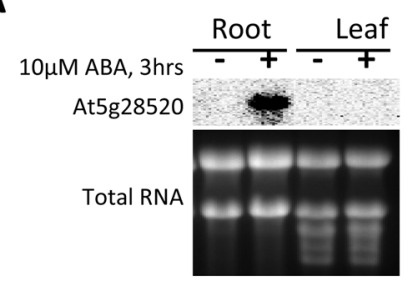

B

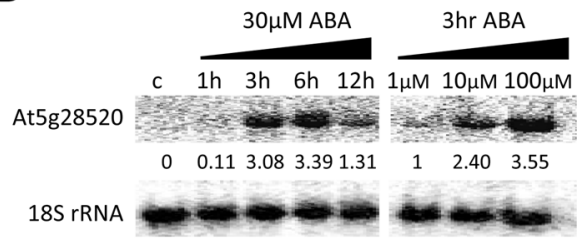

$18 \mathrm{~S}$ rRNA

C

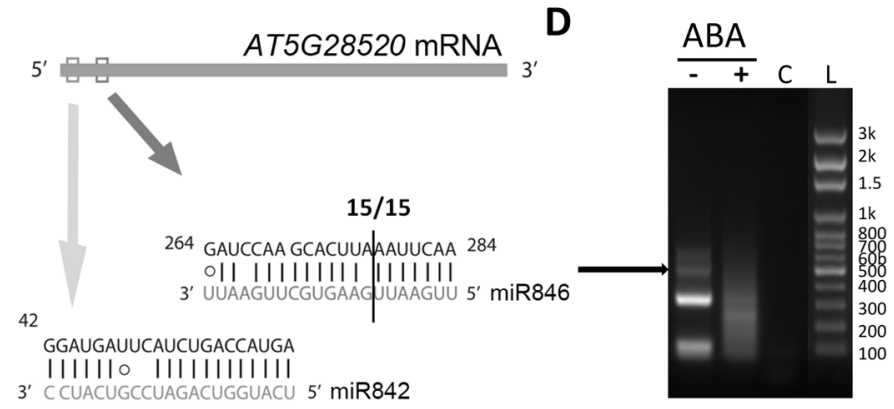

Fig. 4. A potential target of miR842 and miR846 is up-regulated by ABA

(A) Northern blot showing AT5G28520 mRNA in roots or leaves of Col-0 seedlings treated with water or $10 \mu \mathrm{M}$ ABA. EtBr-stained total RNA served as loading controls. $(B)$ Northern blot of total RNA from roots treated by ABA with increasing treatment time or dosage.

Numbers below the bands are relative to signal strength of band from $1 \mu \mathrm{M}$ ABA for $3 \mathrm{hr}$ sample; all samples were normalized internally to band intensity against $18 \mathrm{~S}$ rRNA signal. (C) A cartoon illustrating the complementary sites of miR842 and miR846 on At5g28520 mRNA. The cleavage site detected by modified $5^{\prime}$-RACE is indicated with the black line. The "15/15" label represents the number of sequenced independent clones mapping to the position marked (100\%). (D) Amplicons from modified 5'-RACE on AT5G28520mRNA in roots treated with or without $30 \mu \mathrm{M}$ ABA for $3 \mathrm{hr}$. C, modified $5^{\prime}$-RACE negative control lane. L, 100 bp DNA ladder. Black arrow points to the correct size of the cleavage product mapping to the miR846 complementary site. 
A

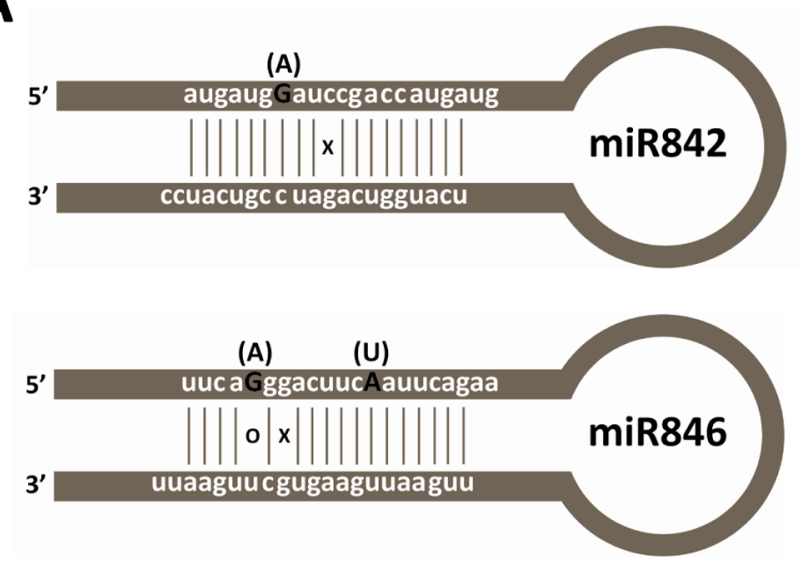

C

D
CAUUCAAGGACUUCAAUUUAA

XM_002888053.1 $\quad x|||||||x|||||||||$ O |

uuaaguucgugaaguuaaguu

Aly-miR846

51

GGAUGAUGGAUCGGACCAUGA

XM_002885914.1
B
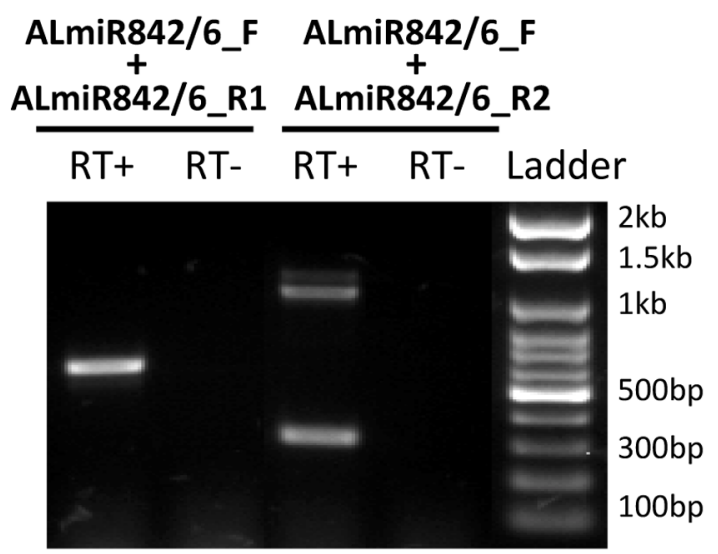

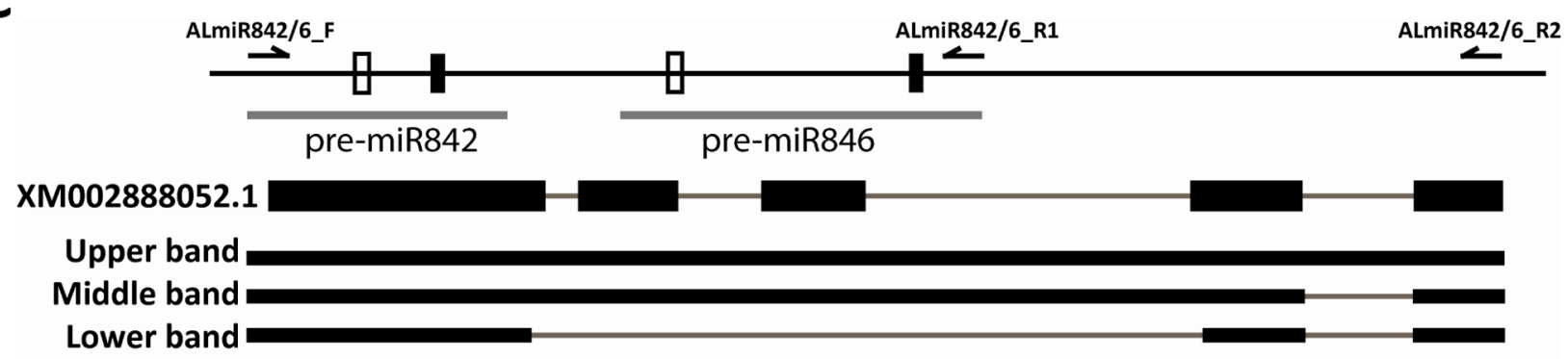

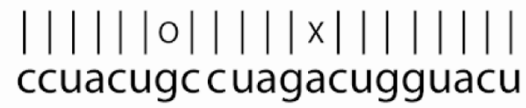

Aly-miR842
483

503

GGAUGAUGGAGCUGACCAUGA

|||||o|||x||||||||||

ccuacugc cuagacugguacu

Aly-miR842

273

293

GAUUCAAGGAAUUCAAUUC AA

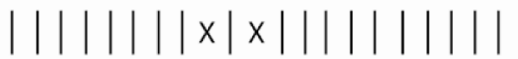

uuaaguucgugaaguuaaguu

Aly-miR846

Fig. 5. miR842, miR846 and their potential jacalin target mRNAs in A. lyrata

(A) The miRNA-miRNA* pairing of miR842 and miR846 in A. thaliana and A. lyrata.

Vertical lines between the arms represent perfect matches. "o" represents a G:U wobble. X represents a mismatch. Letters in brackets represent the substitutions in $A$. thaliana. (B) RT-

PCR using total RNAs from 10-day old $A$. lyrata seedlings. The primers used are described in $(C)$. (C) Illustration of $A$. lyrata genomic region containing $A l y-M I R 842$ and $A l y-$ MIR846, an EST mapping to this region, and three amplicons from RT-PCR in $(B)$, which show similar arrangement as in $A$. thaliana. For the EST and the amplicons, black boxes represent exons and gray lines represent introns. For the A. lyrata MIR842 and MIR846 genomic structure, gray bars (labeled below) represent pre-mir842 or pre-mir846, open vertical rectangles represent miRNA* and black vertical rectangles represent mature miRNA sequences, arrows on the top represent primers used in (B). (D) A. lyrata jacalin homologs 
which carry miR842 and miR846 complementary sites. Vertical lines represent perfect matches. "o" represents a G:U wobble. X represents a mismatch. 